\title{
L'ASIE DU SUD-EST, UN TERRAIN D'INNOVATION PAR LE NUMÉRIQUE POUR LA MOBILITÉ ?
}

Étude de cas à partir des services de taxi à Ho Chi Minh Ville et Kuala Lumpur Manon Eskenazi et Virginie Boutueil

\section{La Découverte | « Réseaux »}

2016/6 n² 200 | pages 61 à 85

ISSN 0751-7971

ISBN 9782707192288

\section{Article disponible en ligne à l'adresse :}

https://www.cairn.info/revue-reseaux-2016-6-page-61.htm

Distribution électronique Cairn.info pour La Découverte.

(C) La Découverte. Tous droits réservés pour tous pays.

La reproduction ou représentation de cet article, notamment par photocopie, n'est autorisée que dans les limites des conditions générales d'utilisation du site ou, le cas échéant, des conditions générales de la licence souscrite par votre établissement. Toute autre reproduction ou représentation, en tout ou partie, sous quelque forme et de quelque manière que ce soit, est interdite sauf accord préalable et écrit de l'éditeur, en dehors des cas prévus par la législation en vigueur en France. Il est précisé que son stockage dans une base de données est également interdit. 


\section{L'ASIE DU SUD-EST, UN TERRAIN D'INNOVATION PAR LE NUMÉRIQUE POUR LA MOBILITÉ?}

Étude de cas à partir des services de taxi à Ho Chi Minh Ville et Kuala Lumpur

Manon ESKENAZI

Virginie BOUTUEIL 


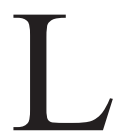

es technologies de l'information et de la communication (TIC) reconfigurent les modalités d'interaction entre individus (Beauchamps et Martin-Brelot, 2014) ainsi que les schémas de mobilité individuelle (Rallet et al., 2009). Elles sont également à l'origine d'une transformation de services de mobilité traditionnels et de la naissance de nouveaux services.

Depuis soixante ans, l'Asie du Sud-Est connaît une croissance urbaine et économique rapide, qui s'accompagne d'une transformation des systèmes de mobilité urbains. Là où la marche constituait historiquement la base de la mobilité, on observe dorénavant une cohabitation plus ou moins régulée, plus ou moins harmonieuse selon les villes, de modes de transport variés (Allaire, 2006). La hausse de la motorisation individuelle à 2 ou 4 roues est un phénomène généralisé et massif dans les villes de la région (Hook et Replogle, 1996), certaines pouvant même être qualifiées de « dépendantes à la motocyclette » (Nguyen et al., 2013). Les problèmes sociétaux majeurs engendrés par cette transformation rapide des systèmes de mobilité - en termes de congestion, d'atteinte à l'environnement et à la santé publique - conduisent les pouvoirs publics dans plusieurs villes à faire de la promotion des modes alternatifs aux modes motorisés individuels un domaine prioritaire de leur action. La région étant par ailleurs le théâtre d'une diffusion rapide du smartphone (Ericsson, 2014), la question se pose du rôle que les applications mobiles sont susceptibles de jouer dans l'inflexion des trajectoires de transformation des systèmes de mobilité dans ces villes.

En prenant pour objet d'analyse le secteur du taxi en Asie du Sud-Est, cette recherche exploratoire propose d'examiner comment l'essor récent des applications mobiles de réservation de taxi affecte les relations entre les différents acteurs - usagers, producteurs et régulateurs - de ce service de mobilité traditionnel dans un contexte d'évolution rapide des systèmes de mobilité. Cette recherche fait pour première hypothèse que l'information échangée au moyen des applications mobiles augmente le pouvoir des usagers et des chauffeurs de taxi en créant de la valeur ajoutée à leur bénéfice. Elle fait pour seconde hypothèse que le déploiement des applications mobiles dans le secteur du taxi modifie le cadre de l'action publique sur ce secteur et questionne le rôle 
des pouvoirs publics en matière de planification, de régulation et de contrôle. Deux villes ont été sélectionnées pour terrains d'analyse, Kuala Lumpur en Malaisie (KL) et Ho Chi Minh Ville au Vietnam (HCMV), pour illustrer une diversité de situations en matière $i$ ) d'intégration du taxi à la démarche de planification des transports urbains (effective à KL, inexistante à HCMV), ii) de conditions d'accès aux licences de taxi (restreinte aux compagnies à HCMV, ouverte aux chauffeurs individuels à $\mathrm{KL}$ ), et iii) de présence d'un secteur informel concurrent (tolérée à HCMV, limitée à KL). Cet article se concentre sur les applications mobiles natives d'Asie du Sud-Est, en particulier l'application GrabTaxi ${ }^{1}$ en raison de son choix de travailler avec les chauffeurs de taxi licenciés ${ }^{2}$. La start-up malaisienne s'est adaptée à différents marchés locaux, proposant une médiation entre les clients et les taxis-voiture, mais travaillant également avec les chauffeurs de mototaxis dans les villes où ce mode de transport s'est fortement développé, comme à HCMV.

L'analyse présentée ici s'inscrit dans le cadre de travaux de recherche plus larges sur les systèmes de mobilité en Asie du Sud-Est ayant mobilisé une importante revue de littérature scientifique dont cet article exploite une partie. L'analyse des jeux des acteurs (usagers, producteurs et régulateurs des services de taxi, fournisseurs d'applications mobiles de réservation) s'appuie sur : $i$ ) une revue des documents de planification des transports urbains, ii) une revue de la presse anglophone généraliste et spécialisée locale (e27, Five Stars and a Moon, Tech in Asia, Thanh Nien News, The Jakarta Post, The Malaysian Insider, Tuoi Tre News) et internationale (Nikkei Asian Review, Tech Crunch, Tech Times), et iii) une enquête de terrain réalisée en juin 2015 à KL et HCMV. Des entretiens semi-directifs ont été conduits auprès de deux catégories d'acteurs, chacune ayant fait l'objet d'une grille d'entretien spécifique :

- Six experts issus du monde universitaire et travaillant en lien avec les pouvoirs publics sur les questions d'urbanisme et de transport au Vietnam et en Malaisie ont été interrogés dans le but d'appréhender la place du taxi dans la mobilité urbaine locale et les stratégies politiques relatives aux nouveaux

1. Créée en 2012 en Malaisie sous le nom MyTeksi, l'application était encore connue sous ce nom au moment de notre étude. Elle a depuis adopté le nom simplifié de Grab.

2. Contrairement à GrabTaxi, Uber travaille essentiellement avec des chauffeurs de véhicules de tourisme, profession dotée d'une licence plus accessible que la licence de taxi. Nous ne nous intéressons pas dans cet article au service GrabCar, développé en complément de GrabTaxi, qui permet de réserver un véhicule de tourisme avec chauffeur (VTC) selon des modalités proches de celles du service qu'Uber propose. 
acteurs du marché (cf. tableau 1 en annexe). Les entretiens avec les experts ont été réalisés en français ou en anglais. Les éléments d'analyse tirés de ces entretiens sont repérés par la mention [entretien expert].

- Huit chauffeurs malaisiens et vietnamiens de taxi ont été interrogés dans le but d'évaluer leur familiarité et leur appétence pour les nouveaux outils numériques en appui à leur activité. Certains entretiens avec les chauffeurs ont été réalisés à l'occasion de trajets tarifés et ont permis de mieux appréhender les enjeux de la mobilité en (moto)taxi dans les deux villes. Tous les entretiens avec les chauffeurs ont été réalisés en anglais.

Dans la première partie de cet article, nous mettons en lumière les spécificités des industries du taxi à Ho Chi Minh Ville et Kuala Lumpur et nous analysons la dynamique de déploiement des applications mobiles de réservation de taxi dans les deux capitales. Dans un deuxième temps, nous examinons comment ces applications mobiles confèrent un pouvoir accru aux usagers et aux chauffeurs de taxi. Enfin, nous examinons la façon dont elles questionnent le rôle des pouvoirs publics en matière de planification, de régulation et de contrôle du secteur.

\section{LE TAXI EN ASIE DU SUD-EST : DYNAMIQUE DE DÉVELOPPEMENT D'UN TRANSPORT DE NICHE ET RÔLE DES APPLICATIONS MOBILES}

À Ho Chi Minh Ville, le transport en commun repose essentiellement sur le bus - la première ligne de métro est prévue pour 2020 - et celui-ci ne représente que $7 \%$ des déplacements quotidiens dans la ville (Mouhot, 2013). La faible qualité de service des bus (en termes d'amplitude horaire, de desserte, de fiabilité, de confort, etc.), combinée au développement de la classe moyenne, favorise l'essor du transport individuel motorisé. Toutefois, la voiture restant un bien de luxe, c'est la motocyclette qui tient le rôle de moyen de transport dominant. Bien que plus développé à Kuala Lumpur qu'à Ho Chi Minh Ville, avec un réseau ferré qui dessert à la fois la ville et son aire urbaine et une fréquentation en hausse, le transport en commun y reste peu attractif et c'est la voiture qui assure la majorité des déplacements de la capitale malaisienne.

Dans les deux villes, le taxi fait figure de solution alternative à la mobilité motorisée individuelle pour les déplacements occasionnels des résidents, notamment pour motifs professionnels et de loisirs. Il est également un maillon essentiel de la mobilité touristique (Eskenazi, 2015), constituant parfois 
le seul moyen de transport desservant des zones d'intérêt peu ou pas desservies en transport en commun. À Kuala Lumpur et Ho Chi Minh Ville, la part modale du taxi se situe entre $1 \%$ et $3 \%$ des déplacements (Onn et al., 2014 ; Mouhot, 2013). L'industrie du taxi dans ces deux villes constitue donc une niche, confrontée à divers défis pour son développement. Non subventionnés, ces services proposent des tarifs (régulés) élevés par rapport au pouvoir d'achat de la population locale et demeurent de ce fait inaccessibles à une part significative de la population pour des besoins de transport porte-à-porte (Darbéra, 2010). La qualité et la sécurité variables des services de taxi constituent également des défis partagés par les services de taxi des deux villes (Eskenazi, 2015).

Si l'exploitation et l'usage des services de taxi à Ho Chi Minh Ville et Kuala Lumpur présentent des similitudes, la place de ces services dans l'offre de mobilité urbaine et dans l'organisation institutionnelle du transport diffère d'une ville à l'autre. Les analyses qui suivent éclairent deux contextes locaux distincts pour ce qui concerne les conditions de développement et la dynamique d'évolution du service.

\section{Cohabitation du formel et de l'informel dans les services de taxi à Ho Chi Minh Ville}

À Ho Chi Minh Ville, le Département des Transports (DOT) de la ville est en charge de la régulation des services de taxi, ainsi que des autres modes de transport en commun urbains [entretien expert]. Au sein du DOT, les bus et le futur métro sont chacun sous tutelle d'une unité dédiée. Au contraire, les services de taxi, quoique reconnus (et régulés) comme du transport d'intérêt public, ne font pas l'objet d'une tutelle dédiée et sont perçus par les autorités publiques comme un mode de transport opéré par le secteur privé et répondant aux seuls besoins d'une portion limitée (et aisée) de la population [entretien expert]. Bien qu'opérant dans un cadre régulé par le DOT, qui contrôle la qualité via des standards et réglementations, les compagnies et associations de taxis jouissent d'une certaine autonomie dans la gestion du secteur. Le taxi figure par ailleurs de façon très marginale dans les documents de planification des transports de la ville.

$\mathrm{Au}$ Vietnam, les licences de taxi ne sont délivrées qu'à des compagnies de taxi, la délivrance de licences individuelles n'étant pas prévue par la réglementation. Seuls deux statuts sont donc possibles pour les chauffeurs de taxi 
d'Ho Chi Minh Ville : ils peuvent être employés par l'une des 26 compagnies licenciées opérant dans la ville, ou bien ils peuvent être franchisés. Dans ce dernier cas, ils louent alors une licence à une compagnie et utilisent sa marque d'exploitation. Le marché des taxis à Ho Chi Minh Ville est dominé par deux entreprises, Vinasun et Mai Linh. La solide réputation de ces deux entreprises repose sur la bonne qualité de leurs véhicules et de leur service (Nguyen, 2014). Elles ont une forte présence dans la ville et sont une référence pour les touristes et les résidents. Elles entretiennent de bonnes relations avec les autorités publiques, ce qui leur donne un avantage sur leurs concurrentes dans le processus d'octroi de licences (Nguyen, 2014). Ces deux entreprises ont par ailleurs renforcé leur position sur le marché grâce à des partenariats avec des centres commerciaux et hôtels [entretien expert], positionnant des représentants sur des stands ou bornes à l'extérieur des bâtiments pour qu'ils orientent les clients vers les taxis qui attendent en file. Ces partenariats sont généralement exclusifs, offrant une visibilité accrue aux compagnies et facilitant aux chauffeurs la mise en relation avec les clients. Cette visibilité permet de consolider leur réputation en termes de qualité de service. L'utilisation d'un modèle unique de véhicules, systématiquement peints aux couleurs de leur compagnie, permet une reconnaissance immédiate par les clients potentiels des compagnies auxquelles ils font confiance. Parmi les problèmes affectant l'industrie du taxi à Ho Chi Minh Ville, des pratiques frauduleuses sont encore régulièrement rapportées ${ }^{3}$, notamment au détriment des touristes, mais c'est la congestion qui fait figure de principal défi. En l'absence de voies de circulation réservées que les taxis pourraient emprunter, ceux-ci sont pris dans le trafic de la même façon que les véhicules personnels, et circulent à une vitesse moyenne de $8 \mathrm{~km} / \mathrm{h}$ aux heures de pointe (HCMC, 2010). Par conséquent, ils sont perçus comme un moyen de transport peu fiable et très onéreux à ces heures de la journée. Au contraire, les motocyclettes, plus agiles et plus rapides, dominent le trafic.

Les mototaxis, appelés localement xe om, se sont développés à Ho Chi Minh Ville comme un mode de transport agile de faible capacité, à l'instar de ce qui peut être observé dans plusieurs pays d'Asie du Sud-Est. C'est une forme de transport « artisanal », dont la propriété est atomisée et qui nécessite un savoir-faire et une certaine autonomie de la part des opérateurs (Godard,

3. "Tips to avoid scam taxi in downtown Ho Chi Minh City », Tuoi Tre News, publié le 12 décembre 2015, http://tuoitrenews.vn/society/32185/tips-to-avoid-scam-taxi-in-downtownho-chi-minh-city. 
1992). Si le transport artisanal peut être formalisé dans certaines villes de pays en développement via l'octroi de licences - c'est le cas des angkots en Indonésie -, les xe om vietnamiens sont opérés de façon informelle, hors du cadre de la régulation des services de transport par les pouvoirs publics. Des statistiques précises manquent sur ce sujet, mais on observe que le secteur du transport informel est un vivier supplémentaire d'emplois faiblement qualifiés à Ho Chi Minh Ville, où la majorité des chauffeurs de xe om ont un niveau d'éducation peu élevé (Tuan et Babiano, 2013) ${ }^{4}$.

À Ho Chi Minh Ville, les mototaxis sont bien adaptés à l'urbanisme et à la situation de congestion de la voirie : plus agiles que les taxis-voiture, ils peuvent se frayer un chemin à travers les rues étroites et encombrées. Ils servent habituellement les besoins de mobilité de la partie la plus pauvre de la population locale, notamment des étudiants et des travailleurs à faible revenu pour les loisirs et déplacements domicile-travail ou domicile-étude (Tuan et Babiano, 2013). Si le recours à des services de xe om est 4 à 7 fois plus coûteux que l'usage de la motocyclette personnelle ou du bus, il reste $60 \%$ moins coûteux que le recours à des services régulés de taxi. Les chauffeurs de $x e$ om n'utilisant pas de taximètre, le coût d'un trajet est négocié entre le chauffeur et l'usager, introduisant ainsi un facteur d'incertitude dans la relation commerciale. Ainsi, en dépit d'une existence hors de tout cadre de régulation, les xe om sont une composante essentielle du système de mobilité urbaine à Ho Chi Minh Ville, représentant selon Tuan et Babiano (2013) de 5 à $11 \%$ des déplacements en semaine, et répondant notamment aux besoins de mobilité des plus pauvres.

\section{Les services de taxi régulés face au défi de la qualité de service à Kuala Lumpur}

En Malaisie, les services de taxi sont régulés au niveau national par la Land Public Transport Commission (SPAD) depuis 2010 en tant que mode de transport public à part entière. Ils font actuellement l'objet d'importantes transformations dans le cadre d'un plan d'amélioration du service établi par la SPAD

4. L'industrie régulée du taxi est elle-même un vivier significatif d'emplois de faible niveau de qualification dans les pays en développement : on compte 24000 licences à Jakarta, autant à Kuala Lumpur et 25000 à Singapour. Sources : pour Jakarta, « Does Jakarta have too many cabs? », The Jakarta Post, 12 décembre 2011 ; pour Kuala Lumpur, SPAD, 2013 ; pour Singapour, http://www.taxisingapore.com/taxi-companies. 
(SPAD, 2013). Ce plan réglemente les tarifs, les licences et les standards de qualité de service. En tant que mode de transport public, les services de taxi sont identifiés comme solutions aux besoins de mobilité du dernier kilomètre (SPAD, 2013).

Les services de taxi font l'objet d'une classification officielle en quatre catégories correspondant à des qualités d'offre et des segments de demande différents (cf. tableau 2 en annexe). Contrairement au cas du Vietnam, les licences de taxi malaisiennes peuvent être accordées à deux types d'opérateurs, entreprises ou particuliers. Les licences dans les catégories Budget, Premium (catégorie en cours de retrait) et Executive sont majoritairement attribuées à des compagnies. Dans la catégorie TEKS1M, introduite en 2013 par la SPAD pour améliorer la qualité de service des opérateurs de taxi individuels avec des véhicules plus confortables et mieux équipés (SPAD, 2013), les licences sont exclusivement délivrées à des particuliers. Dans l'ensemble, la profusion de licences individuelles tend à rendre plus difficile le contrôle de la qualité du service de taxi par la SPAD.

Les services de taxi malaisiens ont une réputation de piètre fiabilité [entretien expert], laquelle tient en partie à la présence sur le marché de taxis illégaux non équipés de taximètre et non licenciés que les autorités de la ville ont cherché à éradiquer (SPAD, 2013). Les prestations de ces taxis illégaux sont généralement plus onéreuses que les courses au taximètre, et des atteintes à la sécurité des passagers, en particulier des vols, ont été rapportées, comme en témoignent divers forums en ligne dédiés au tourisme. Dans ce contexte, le défaut d'information préalable à la course du client sur la licence, le chauffeur ou le véhicule peut être perçu comme un facteur d'incertitude (sur la fiabilité, le coût, etc.), voire d'insécurité.

\section{La dynamique de déploiement des applications mobiles de réservation de taxi dans les deux capitales}

Les applications mobiles sont en passe de devenir des outils cruciaux d'organisation et de facilitation de la mobilité quotidienne des individus (Widmer, 2014). La question du rôle que sont susceptibles de jouer les applications mobiles de réservation de taxi dans la transformation du secteur en Asie du Sud-Est se pose dans un contexte où la pénétration des smartphones est en forte hausse en Asie du Sud-Est. Ericsson (2014) prévoit ainsi que la région 
comptera 700 millions d'usagers de smartphones en 2019, alors qu'ils étaient 300 millions en 2013. Au Vietnam, où le marché du smartphone est le plus dynamique, $55 \%$ de la population adulte possédait un smartphone en 2015 (We Are Social, 2016), un chiffre similaire à celui du marché malaisien, où le smartphone atteignait $51 \%$ de pénétration en 2014 (Google, 2014).

Les applications de réservation de taxi basées sur les TIC mobiles sont un médium pour mettre en relation les clients et les chauffeurs. Elles se sont généralement développées à l'initiative d'acteurs extérieurs à l'industrie du taxi. En Asie du Sud-Est, le développement de ces applications a été initié par Uber qui, fort de son application rodée au plan international, a commencé à développer dans la région son activité positionnée sur le marché de la réservation de véhicule de tourisme avec chauffeur (VTC). Des start-up locales, telles que Grab, se sont créées par la suite pour concurrencer l'entreprise américaine au moyen d'applications mobiles de réservation de taxi développées localement. Là où Uber est susceptible de travailler non seulement avec des chauffeurs professionnels (taxi ou VTC), mais aussi avec des chauffeurs sans licence (ni de taxi ni de VTC) via son service UberPop, les applications développées localement mettent l'accent sur la mise en relation des clients avec des chauffeurs de taxi dûment licenciés. À l'été 2016, Grab était présente dans 30 villes d'Asie du Sud-Est (contre 16 pour Uber) et revendiquait 19 millions de téléchargements de l'application et 350000 chauffeurs ${ }^{5}$.

Uber et Grab ont rencontré la résistance des pouvoirs publics et du cadre réglementaire en vigueur quand ils sont entrés sur le marché vietnamien. Les deux entreprises ont choisi de ne pas s'enregistrer comme opérateurs de transport, mais comme sociétés de prestation de services informatiques, mettant en avant leur rôle de plateforme de mise en relation entre clients potentiels et chauffeurs ${ }^{6}$. Par conséquent, le cadre législatif n'a réglementé les services qu'a posteriori. À Ho Chi Minh Ville, les compagnies de taxi et les pouvoirs publics n'ont pas, au moins dans un premier temps, appréhendé et intégré dans leurs champs respectifs d'activité les changements apportés par les

5. «Grab CEO: Didi victory shows we can beat Uber in Southeast Asia », Tech Crunch, publié le 31 juillet 2016, https://techcrunch.com/2016/07/31/grab-ceo-didi-victory-shows-we-canbeat-uber-in-southeast-asia (consulté le 10 novembre 2016).

6. "Uber ordered to respect Vietnam laws when running cab-hailing service ", Thanh Nien News, publié le 22 mars 2015, http://www.thanhniennews.com/business/uber-ordered-to-respect-vietnam-laws-when-running-cabhailing-service-40073.html 
applications mobiles ${ }^{7}$. Ce sont les clients et les chauffeurs qui ont poussé à l'expérimentation de ces nouveaux outils. Au contraire, en Malaisie, le gouvernement national a fait de l'innovation un élément crucial de la stratégie de Kuala Lumpur pour se hisser au rang des « villes globales » (Bunnell et al., 2002). Ainsi, en dépit d'un cadre réglementaire fort, le contexte sociotechnique en Malaisie est favorable au développement de l'usage des TIC dans le domaine de la mobilité [entretien expert]. À ce titre, la SPAD a intégré Grab dans sa stratégie de transformation du taxi, avec la mise en œuvre de partenariats pour améliorer les services de taxi pour la mobilité des touristes, notamment par la mise en place de bornes de réservation GrabTaxi dans les lieux touristiques (Visit KL, 2014).

\section{LA REVENDICATION D'UNE «MISSION SOCIALE » DES APPLICATIONS DE RÉSERVATION DE TAXI, AU SERVICE DE L'EMPOWERMENT DES USAGERS ET DES CHAUFFEURS}

Le président-directeur général de Grab, développeur de GrabTaxi et GrabBike, décrit ses applications comme ayant une «mission sociale $»^{8}$. En effet, elles sont conçues pour surmonter simultanément deux difficultés majeures : i) la quête du prochain client pour les chauffeurs, et ii) l'incertitude des usagers quant à la disponibilité et à la qualité du service de taxi. Les applications mobiles de réservation de taxi se positionnent donc comme les outils d'une transformation de l'opération et de l'usage des services de taxi dans une dynamique bottom-up, en partant des problèmes des chauffeurs et des clients. À ce titre, et au regard de la littérature scientifique consacrée à ce concept, ces applications mobiles peuvent donc être qualifiées d' " innovations sociales », au sens où elles apportent des solutions en réponse à des besoins exprimés par la société (De Muro et al., 2007).

7. « Meanwhile, in Vietnam: Uber vs GrabTaxi vs Vinasun vs Mai Linh vs EasyTaxi », TechinAsia.com, publié le 4 décembre 2014, https://www.techinasia.com/vietnam-uber-grabtaxi-vinasun-mai-linh-easytaxi/ (consulté le 8 décembre 2014).

8. "My first venture was at 11, says Anthony Tan, who traded comics then », e27, 10 février 2014, https://e27.co/first-venture-11-says-anthony-tan-traded-comics/ 


\section{L'intensification et la diversification de l'activité des chauffeurs par la facilitation de la mise en relation de l'offre et de la demande}

Le fondateur de Go-Jek, une application mobile de réservation de mototaxi indonésienne, a déclaré lors du New Cities Summit 2015 que le problème des pays en développement n'était pas le chômage, mais le sous-emploi. Le diagnostic établi par la SPAD dans son plan de transformation du taxi (SPAD, 2013) est à cet égard éloquent : les chauffeurs de taxi à Kuala Lumpur circuleraient à vide à hauteur de $60 \%$ de leur temps d'activité. De la même façon, Tuan et Babiano (2013) estiment qu'à Ho Chi Minh Ville, la moitié du temps d'activité des conducteurs de mototaxis informels serait consacrée à l'attente des clients.

La littérature scientifique ne positionne pas les applications mobiles de réservation de taxi comme une transformation radicale des pratiques de réservation de taxi : elles sont le prolongement numérique de pratiques anciennes pour héler un taxi dans la rue, dont la réservation par téléphone fut la première (r)évolution (Perez et Armand, 2016). Malgré la démocratisation du téléphone portable et des outils numériques, les applications mobiles ne sont pas majoritaires dans les pratiques de réservation, notamment dans des pays comme le Vietnam où l'usage du smartphone est encore en développement [entretien expert]. Pourtant, elles sont susceptibles d'augmenter le potentiel d'appariement entre une offre et une demande diffuses de mobilité (Adoue, 2014), et par conséquent la productivité des services de taxi.

Dans les villes d'Asie du Sud-Est, les techniques traditionnelles de mise en relation entre les chauffeurs de taxi et leurs clients sont la réservation par téléphone, la rencontre en des points dédiés (bornes ou stands aux abords des hôtels et centres commerciaux, par exemple) et, bien sûr, la maraude (SPAD, 2013). Selon les villes, les techniques traditionnelles rencontrent des succès divers. En particulier, la performance de la réservation par téléphone diffère fortement entre Ho Chi Minh Ville et Kuala Lumpur, ce qui s'explique en partie par les différences dans l'organisation du secteur du taxi dans ces deux villes. À Ho Chi Minh Ville, les experts interrogés considèrent que le système téléphonique de réservation fonctionne bien, et cette technique prédomine sur l'utilisation des applications mobiles. Les licences de taxi n'étant attribuées qu'à des compagnies, celles-ci utilisent leurs centrales d'appel dédiées pour répartir les courses entre les voitures, ce qui leur permet de garder une relation directe avec le client ainsi qu'avec le chauffeur, salarié de la compagnie, et 
d'assurer un suivi de la course. Au contraire à Kuala Lumpur, la SPAD estime que jusqu'à $60 \%$ des courses réservées par téléphone ne sont pas honorées, de nombreux chauffeurs renonçant aux courses les éloignant trop de leur localisation au moment de la réservation (SPAD, 2013). Dans un contexte où la plupart des chauffeurs de taxi sont des individuels opérant à leur propre compte, la répartition des courses par les centrales d'appel semble d'une moindre efficacité en raison de l'absence de lien de subordination entre les centrales et les chauffeurs et de la faiblesse des outils dont disposent les centrales pour assurer un suivi des courses. On notera qu'outre une facilitation de la mise en relation en temps réel entre les chauffeurs et leurs clients potentiels, les applications mobiles augmentent la probabilité de réalisation effective de la course en relayant la demande du client à un chauffeur sélectionné selon une logique de proximité (permise par la géolocalisation en temps réel) et par la redirection, en cas de déclin de la course par le chauffeur sélectionné, vers un autre chauffeur dans le périmètre?.

Dans le secteur informel des mototaxis, le problème du sous-emploi est accentué par un déficit d'expertise de conducteurs qui guettent généralement leurs clients en stationnement, et sans disposer de caractéristiques reconnaissables - tenue vestimentaire, véhicules floqués - permettant leur identification par des clients potentiels dans la rue. À Jakarta, Go-Jek a entrepris de traiter cette question à l'aide d'une application mobile, et pour ce faire a équipé de smartphones les chauffeurs de mototaxis. L'ambition de telles applications n'est pas seulement de permettre la pénétration du marché du taxi par les acteurs du secteur informel, mais si possible de l'étendre au-delà de ses limites de niche. Le secteur informel des mototaxis s'y prête d'autant plus qu'il se caractérise par une forte capacité d'adaptation (Bessone et Cusset, 1989). L'application Go-Jek a non seulement permis aux conducteurs d'étendre leur zone de chalandise, mais leur a également fourni des systèmes de navigation pour optimiser leur course. Les clients bénéficient également de ces améliorations, par un accès facilité au service et des conditions plus efficaces de réalisation des trajets.

En complément à leur activité principale de transport de personnes, Go-Jek en Indonésie et Grab au Vietnam offrent à leurs clients des services de livraison.

9. « Cabbie shares his thoughts on GrabTaxi and the driving life... », Five Stars and a Moon, 30 avril 2014, http://www.fivestarsandamoon.com/cabbie-shares-his-thoughts-on-grab-taxiand-the-driving-1. 
Les chauffeurs affiliés à ces applications mobiles sont ainsi amenés à diversifier leur activité et par conséquent leurs sources de revenus. La diversification des activités des chauffeurs peut être facilement mise en œuvre dans l'industrie du mototaxi informel, où le métier de chauffeur n'est, par construction, pas réglementé. On observe cette dynamique de diversification des activités de mototaxi à Ho Chi Minh City notamment. Dans le secteur formel au contraire, la question se pose de la compatibilité de l'hybridation du transport de passagers et du transport de marchandises avec le cadre réglementaire relatif à la profession de chauffeur de taxi.

\section{La réduction de l'incertitude pour l'usager et l'amélioration de la qualité de service perçue}

Les applications mobiles ne constituent pas seulement des outils efficaces de mise en relation entre chauffeurs et clients. Elles enrichissent également le service de réservation de taxi de nouvelles fonctions dont les techniques traditionnelles sont privées et qui contribuent à réduire l'incertitude de l'usager du taxi sur le déroulement de sa course, de sa réservation jusqu'à son achèvement.

La notion d'incertitude est inhérente à la mobilité des personnes, car l'individu traverse des espaces et est contraint par des aléas qu'il ne maitrise pas (Salles, 2015). Lorsqu'un client hèle un taxi dans la rue ou commande une course par téléphone, il est exposé à une incertitude d'autant plus forte que les inconnues portent également sur l'identité du chauffeur et sa fiabilité, le prix de la course à l'arrivée, etc. Il n'a accès ni à l'information sur le service a priori (sur le chauffeur, la compagnie, etc.) ni à l'information en temps réel qui lui permettrait de mieux maitriser les conditions de réalisation de sa course (sur l'état de la circulation, par exemple). Les outils numériques améliorent sensiblement l'accès des usagers aux informations en temps réel sur les moyens de transport (Sajous et al., 2015) et leur permettent, par un enrichissement des fonctions proposées, de mieux maîtriser leur mobilité et de développer de nouvelles pratiques pour remplacer des pratiques jugées insatisfaisantes (Rocci, 2007). Dans le cas du taxi, les applications mobiles de réservation offrent aux usagers des moyens de contrôle et d'optimisation de leur déplacement dont ils ne disposaient pas auparavant. En effet, ces applications mobiles accompagnent l'usager $i$ ) en amont de la course, en les informant sur le chauffeur, sa géolocalisation en temps réel et le temps d'attente prévisible, et en proposant des estimations du coût de la course et de sa durée, ii) pendant la course, avec une 
fonction de suivi de la course par GPS ainsi qu'un dispositif de sécurité qui permet d'alerter les proches et la police en cas de problème, et iii) en aval de la course, en permettant l'évaluation de la prestation.

À Kuala Lumpur, l'autorité de régulation du transport reconnaît ouvertement l'insuffisance de la qualité de service du secteur du taxi malaisien (SPAD, 2013). Le fondateur de Grab revendique d'avoir conçu son application mobile pour apporter une réponse à ce problème récurrent ${ }^{10}$. Sur un fondement similaire, les pouvoirs publics malaisiens ont développé l'application mobile Meter On qui permet à l'usager de vérifier les informations relatives au chauffeur de taxi - validité de la licence, historique des plaintes éventuelles, etc. - et de contrôler les conditions de réalisation de la course en temps réel. Grab propose des fonctionnalités additionnelles d'information sur le chauffeur (évaluation par les clients) et d'anticipation sur les caractéristiques du trajet (calcul d'itinéraire, estimation de durée et de tarif) en amont de la course.

Les applications mobiles de réservation de taxi recueillant des données sur les usagers, les chauffeurs et les courses, elles ont pu être décrites comme « des yeux et des oreilles électroniques » (Berthoud 2002, p. 159). La collecte de données, bien que généralement perçue comme présentant des risques pour le respect de la vie privée des individus, peut également être porteuse d'opportunités (Widmer et al., 2014). Pour les usagers des taxis malaisiens, les applications mobiles de réservation peuvent avoir une influence positive en termes de confiance dans le cadre de la relation client-chauffeur. Elles réduisent la vulnérabilité ressentie par l'usager en lui fournissant un outil de vérification de certaines informations relatives au chauffeur et de contrôle des conditions de réalisation de la course. Alors que des risques persistent pour la sécurité des usagers dans les services de taxi malaisiens (SPAD, 2013), les applications mobiles de réservation facilitent également la transmission d'informations en temps réel sur l'itinéraire de l'usager à sa famille, et l'avertissement de la police en cas d'atteinte à la sécurité de l'usager pendant la course.

10. «My first venture was at 11, says Anthony Tan, who traded comics then » $e 27,10$ février 2014, https://e27.co/first-venture-11-says-anthony-tan-traded-comics/ 


\section{LA MODIFICATION DE L'ÉQUILIBRE DES POUVOIRS ET LA REMISE EN CAUSE DU RÔLE DES POUVOIRS PUBLICS DANS LA RÉGULATION DU SECTEUR DU TAXI}

Même si leur marché reste encore limité par la diffusion progressive du smartphone, les applications mobiles de réservation de taxi sont susceptibles de modifier l'équilibre des pouvoirs dans le secteur en en favorisant la transformation par des processus bottom-up (Louvet et al., 2013). En organisant l'échange d'informations entre les opérateurs et les clients au profit de leur montée en autonomie conjointe (Louvet et al., 2013), les applications de réservation modifient plus en profondeur la culture du taxi. En particulier, elles accroissent les attentes des clients, et leur confèrent de nouveaux rôles (d'évaluation des courses et des chauffeurs, par exemple). À l'instar des TIC dans d'autres secteurs de la mobilité, elles contribuent à faire des usagers des acteurs à part entière du service qui leur est rendu (Flichy, 2016). La question se pose alors du positionnement des acteurs traditionnels, en appui ou en résistance à ces innovations qui modifient les équilibres antérieurs des pouvoirs. Louvet et al. (2013) soulignent le fait que les structures préexistantes peuvent « bloquer » le développement de l'innovation par des verrous liés aux réglementations en vigueur ou par des résistances plus diffuses au changement. Des soutiens contradictoires peuvent se faire jour entre, d'une part, des pouvoirs publics défendant l'industrie régulée du taxi et, d'autre part, des populations locales apportant leur soutien aux nouveaux entrants qu'elles jugent plus performants. Amar (2004) relève cependant que, pour favoriser un développement durable des systèmes de mobilité, les pouvoirs publics ont un « devoir d'innovation » qui les oblige à intégrer les innovations émergentes dans la régulation des mobilités.

\section{Un transfert de la responsabilité de contrôle de la qualité de service du taxi vers les usagers et les opérateurs?}

Au-delà des informations fournies aux usagers en préparation et en cours de trajet, les applications mobiles de réservation de taxi leur donnent un nouveau levier d'influence sur le service par le biais de dispositifs de notation et de commentaires. Les utilisateurs de Grab, par exemple, peuvent évaluer les chauffeurs et commenter les courses. Les mauvaises critiques peuvent conduire à une exclusion des chauffeurs incriminés des bénéfices de l'application, les privant ainsi d'une source de revenus complémentaires. Les notations et commentaires sont donc un outil de contrôle utile non seulement pour 
les usagers, mais aussi pour les opérateurs de services. Ils constituent un vecteur d'amélioration de la qualité du service. Les plaintes concernant les services de taxi à Kuala Lumpur concernent principalement le comportement des chauffeurs et les caractéristiques des véhicules (SPAD, 2013). Les standards de qualité associés au processus d'attribution des licences étant faibles, de l'aveu même des pouvoirs publics (SPAD, 2013), les applications mobiles de réservation établissent des référentiels aux exigences accrues qui s'appliquent aux chauffeurs qui leur sont affiliés. La responsabilité du contrôle de la qualité de service, traditionnellement attribuée aux opérateurs de transport et aux autorités dans le domaine du transport public, est reportée sur les applications mobiles et repose sur les contributions des usagers.

\section{Une solution palliative de structuration du secteur informel ?}

Du transport informel apparaît dans les villes où le transport formel organisé et régulé par la puissance publique ne répond pas aux besoins d'une grande partie de la population (Bordeleau, 2015). Dans certaines villes en développement, le transport public repose exclusivement sur le secteur informel (UN-HABITAT, 2013). Celui-ci peut prendre la forme d'un transport collectif de moyenne à grande capacité - notamment en Afrique subsaharienne - ou celle d'un transport de faible (autorickshaws indiens) voire très faible (xe om vietnamien) capacité ; plusieurs modes peuvent émerger au sein d'une même ville, combinant des véhicules à moyenne capacité, comme des minibus, et des véhicules à faible capacité (rickshaws, mototaxis). Dans les villes des pays en développement, les modes informels de faible capacité ne semblent pas tant concurrencer les modes de transport collectif formels que leur être complémentaires, dans la mesure où ils desservent des trajets relativement courts d'une part, où ils sont plus flexibles en termes d'amplitude horaire et de couverture du territoire d'autre part (Rahman, 2013). Le secteur informel présente généralement une forme d'organisation du travail éloignée de celle du secteur formel (Bessone et Cusset, 1989) : les unités de travail sont petites, voire individuelles, et nombreuses; le marché est ouvert à la concurrence; le travail est non protégé ; les activités sont souvent artisanales ou familiales, et nécessitent peu de ressources.

Les problèmes de pollution, de congestion et de sécurité routière liés au transport informel ont amené un nombre croissant de chercheurs à étudier les modalités et les impacts potentiels d'une régulation de ces services (Schalekamp et McLachlan, 2016; Lomme et Vircoulon, 2006 ; Kaf et al., 2007). La régulation 
du transport informel dans des villes où il assure une part significative des déplacements offre une opportunité de mieux contrôler la qualité du service via l'octroi de licences associé à la mise en place de standards de qualité décidés par la puissance publique. De tels dispositifs sont cependant difficiles à mettre en œuvre pour les pouvoirs publics et se heurtent parfois à la réticence des acteurs ciblés par la régulation (Keutcheu, 2015). De plus, en augmentant les coûts pour les opérateurs, la régulation fait courir le risque d'une augmentation des tarifs pour l'usager et par conséquent d'une moindre accessibilité aux services de transport pour les catégories les plus défavorisées de la population. À défaut de régulation par les pouvoirs publics, des processus d'auto-organisation des transports informels peuvent être observés dans certains contextes. Dans les villes africaines, il n'est pas rare de voir les petits opérateurs de transport informel se réunir en coopératives ou en associations pour se développer, et même contrôler certaines routes ou certains territoires (Cervero et Golub, 2007). Ainsi à Lomé, les conducteurs de mototaxis s'organisent autour de stations et se constituent également en syndicats (Guézéré, 2012).

Au Vietnam, la faible part modale des déplacements en mototaxi semble justifier l'absence de régulation de ce mode qui sert principalement les besoins en mobilité des plus pauvres. Aucun réel processus d'auto-organisation ne semble par ailleurs toucher les xe om vietnamiens : la structure du secteur est horizontale et artisanale, avec une multitude d'opérateurs-chauffeurs travaillant à leur compte. Les rares sociétés de mototaxi se limitent à de petites entreprises disposant de peu de pouvoir pour fédérer les opérateurs (Tuan et Babiano, 2013). Les opérateurs de mototaxi ne disposent pas non plus d'associations pour les représenter et engager un dialogue avec les autorités, contrairement au secteur formel du taxi. De par leur cœur de métier (taxi) et leur statut informel, les chauffeurs de xe om sont donc à double titre exclus de la planification des transports par les pouvoirs publics, qui ont opté pour une politique de tolérance et de laissez-faire à leur égard.

Les innovations sociales que sont les applications mobiles peuvent jouer un rôle dans la structuration du secteur du mototaxi. Sur le modèle de Go-Jek en Indonésie, la compagnie Grab au Vietnam a équipé de smartphones des chauffeurs de xe om afin de leur permettre d'utiliser son application GrabBike. Outre la facilitation de la mise en relation et du processus de réservation, les applications mobiles proposent d'autres fonctionnalités qui pourraient contribuer à structurer le secteur. Quand, dans le secteur informel traditionnel, le prix des courses est fixé par négociation entre le chauffeur et le client, les 
clients locaux disposent de références pour fixer des prix raisonnables tandis que les touristes sont moins outillés pour négocier. Tuan et Babiano (2013) soulignent le fait que l'introduction de compteurs pourrait contourner ce système de négociation qui se traduit par une incertitude forte pour le client sur le prix de la course. GrabBike n'a pas encore mis en œuvre la fonctionnalité de compteur dans son application ; pourtant, celle-ci contribuerait à structurer l'industrie en rendant le processus de fixation des prix plus transparent. La start-up indonésienne Go-Jek a utilisé son application pour rassembler les chauffeurs sous son pavillon et unifier le processus de fixation des prix, en échange d'une plus grande visibilité et d'une augmentation des revenus. Introduire des éléments standardisés, tels que des tarifs et également une formation à la sécurité routière - en réponse aux problèmes sérieux que rencontrent de nombreuses villes disposant de services informels de mototaxi -, contribue à asseoir la légitimité et accroître le pouvoir d'influence des opérateurs de mototaxi dans leur dialogue avec les pouvoirs publics (Friedberg, 1992).

\section{CONCLUSION}

Composants traditionnels des systèmes de mobilité urbaine dans les villes des pays en développement, les services de taxi répondent à des besoins spécifiques pour les loisirs ou la mobilité professionnelle et se positionnent en complémentarité avec les services de transport public de masse pour le dernier kilomètre. Ils sont aussi une source importante d'emplois peu qualifiés dans des pays où le niveau d'éducation reste généralement faible. Pourtant, notre analyse a révélé que, dans les deux villes étudiées, les services de taxi sont considérés par les usagers et les pouvoirs publics comme un mode de transport aux performances médiocres en termes de couverture géographique, fiabilité horaire, coût, qualité de service, etc. Les réponses apportées à ces critiques diffèrent dans les deux villes, sous l'influence des autorités publiques d'une part, du secteur privé d'autre part. À Kuala Lumpur, les autorités de transport ont agi sur le processus de délivrance des licences pour améliorer le service, en introduisant sur un segment d'offre critique - les taxis individuels - une nouvelle catégorie de licence assortie de contraintes renforcées. À Ho Chi Minh Ville, le secteur informel s'est développé spontanément sous la forme de services de mototaxi pour offrir une alternative aux populations à faible revenu.

Dans un contexte où les smartphones pénètrent rapidement les marchés du Sud-Est asiatique, les applications mobiles de réservation de taxi peuvent 
servir de support au renouveau des services de taxi. Le déploiement de ces applications peut s'appuyer sur un cadre institutionnel favorable, comme c'est le cas en Malaisie où la SPAD les a prises en compte dans le processus de planification des transports publics. En l'absence d'un cadre institutionnel favorable, comme c'est le cas à Ho Chi Minh Ville notamment, le déploiement des applications mobiles de réservation peut néanmoins tirer parti de la présence d'un secteur informel développé, lequel est par construction affranchi des contraintes et inerties éventuelles du cadre réglementaire applicable aux transports formels. Les bénéfices immédiats de ces applications tiennent essentiellement au processus d'empowerment des usagers et des chauffeurs qu'elles sous-tendent et qui se manifeste par une prise de contrôle accrue des premiers sur leur mobilité et des seconds sur leur activité. Aux chauffeurs, ces applications permettent en effet d'étendre leur zone de chalandise et plus généralement de faciliter la mise en relation avec le client. Aux usagers, ces applications fournissent de nouveaux outils pour assumer un rôle actif dans l'organisation et le bon déroulement de leur mobilité en taxi.

En contrepartie, le déploiement des applications de réservation de taxi interroge sur d'éventuels transferts de responsabilité des pouvoirs publics vers les usagers ou les opérateurs du secteur en matière de planification, de régulation et de contrôle. En matière de contrôle, les dispositifs mis en place par les applications mobiles pour l'évaluation des chauffeurs et le commentaire des courses par les usagers interviennent en relais, quand ce n'est pas en substitution, des efforts de la puissance publique pour contrôler la fiabilité et la sécurité des opérations de taxi. En matière de régulation, les applications mobiles de réservation de taxi semblent pouvoir contribuer à l'auto-organisation, voire l'autorégulation, des services informels (par exemple, sur la qualité, les tarifs, l'hybridation des services) là où la puissance publique ne souhaite pas ou ne parvient pas à réguler. Les applications mobiles semblent même pouvoir s'immiscer dans les fonctions de planification et d'aménagement par le biais de partenariats stratégiques avec les pouvoirs publics permettant notamment la mise en place de bornes de réservation dédiées dans l'espace public.

Cette recherche exploratoire a permis de mettre à jour des besoins de recherche très divers. La structuration du transport informel par les applications mobiles, le report du rôle de contrôle de la qualité de service des pouvoirs publics vers les applications mobiles et les usagers, la remontée d'informations des applications mobiles vers les pouvoirs publics (sur les trafics, les tarifs, la clientèle, etc.), ainsi que l'hybridation des services de (moto)taxi pour les personnes 
et les marchandises (qui plus est dans un contexte de forte croissance du e-commerce ${ }^{11}$ ), sont autant d'enjeux d'action publique qui mériteraient de faire l'objet de recherches plus approfondies. D'autres recherches seront nécessaires pour mieux évaluer, d'une part, les modalités de pénétration des technologies mobiles sur le marché du Sud-Est asiatique (inégalités d'accès à la technologie, etc.) et, d'autre part, l'influence effective de ces technologies sur les pratiques de mobilité, la productivité des services et les bénéfices économiques et sociaux réels pour les chauffeurs. À moyen terme et sur un plan plus macroscopique, une réflexion méthodologique et des analyses empiriques poussées pourront s'avérer utiles à l'évaluation de l'influence des applications mobiles sur la concurrence entre les modes et services de mobilité urbains et des impacts économiques, sociaux et environnementaux des transformations des systèmes de mobilité par le numérique.

11. Le gouvernement vietnamien indique que le chiffre d'affaires de l'e-commerce devrait doubler chaque année au cours de la décennie à venir, https://www.techinasia.com/top15-most-funded-ecommerce-startups-vietnam. 


\section{RÉFÉRENCES}

ADOUE F. (2014), « Les TIC, nouvel Eldorado des politiques de régulation de la mobilité ? Une analyse du discours d'acteurs de l'innovation dans les transports », Géo Regards, n 7, pp. 73-87.

ALLAIRE J. (2006), « Choisir son mode de ville : Formes urbaines et transport dans les villes émergentes », Les Cahiers de GLOBAL CHANGE, n² 21, pp. 66-70.

AMAR G. (2004), Mobilités : éloge de la diversité et devoir d'invention. Paris, Éditions de l'Aube.

BEAUCHAMPS M., MARTIN-BRELOT H. (2014), «M@rsouin : 13 ans de recherche sur les usages numériques. Quelles relations avec la dimension spatiale ?», Netcom, $\mathrm{n}^{\circ}$ 28-3/4, pp. 165-180.

BERTHOUD G. (2002), "L'horizon d'une surveillance omniprésente ? », Revue européenne des sciences sociales. Cahiers Vilfredo Pareto (XL-123), pp. 155-168.

BESSONE M., CUSSET J.-M. (1989), Transports informels en Afrique, en Amérique latine et en Asie du Sud-Est. Analyse comparative, HALSHS. https://halshs.archivesouvertes.fr/halshs-00602126

BORDELEAU B. (2015), « Capsule thématique : le transport informel », http://www. vrm.ca/le-transport-informel/

BUNNELL T., BARTER P. A., MORSHIDI S. (2002), « Kuala Lumpur metropolitan area - A globalizing city-region », Cities, vol. 19, n 5, pp. 357-370.

CERVERO R., GOLUB A. (2007), " Informal transport: a global perspective », Transport Policy, $\mathrm{n}^{\circ}$ 14, pp. 445-457.

DE MURO P., HAMDOUCH A., CAMERON S., MOULAERT F. (2007), « Organisations de la société civile, innovation sociale et gouvernance de la lutte contre la pauvreté dans le Tiers-Monde », Mondes en développement, $\mathrm{n}^{\circ} 139$, pp. 25-42.

ERICSSON (2014), Southeast Asia and Oceania. Ericsson Mobility Report Appendix, p. 8.

ESKENAZI M. (2015), Enjeux et modalités du développement du taxi dans l'offre urbaine de mobilité en Asie du Sud-Est. Le cas de Kuala Lumpur (Malaisie) et Ho-Chi-Minh-Ville (Vietnam), mémoire de Master 2, École d'Urbanisme de Paris, Université Paris-Est, p. 133.

FLICHY P. (2016), « Réflexion sur l'innovation : l'usager et le concepteur », intervention au séminaire innovation du GERI USACT, 20 mai 2016.

FRIEDBERG E. (1992), «Les quatre dimensions de l'action organisée », Revue française de sociologie, $\mathrm{n}^{\circ} 33-4$, pp. 531-557. 
GODARD X. (1992), Mobilité et transport dans les villes en développement, Paris, L'Harmattan, 1992, p. 233.

GOOGLE (2014), 6 Statistics About Malaysian Smartphone Users That You Need To Know, https://plus.google.com/107102605199039331236/posts/eN5BE1A6D7Y

GUEZERE A. (2012), «Territoires des taxis-motos à Lomé : de la pratique quotidienne à la recomposition des espaces urbains et des liens sociaux », Géographie, économie, société, vol. 14, pp. 53-72.

HCMC. (2010), « The current situation and development planning for transportation of Ho Chi Minh City towards $2020 »$, rapport, http://sgtvt.hochiminhcity.gov.vn/hinhanhtintuc/2011-12/duandautu/THUCTRANGQUYHOACH_E[1].pdf.

HOOK W., REPLOGLE M. (1996), « Motorized and non-motorized transport in Asia. Transport system evolution in China, Japan and Indonesia », Land Use Policy, vol. 13, n 1 , pp. 69-84.

KAFFO C., KAMDEN P., TATSABONG B., DIEBO L.-M. (2007). « L'intégration des "moto-taxis" dans le transport public au Cameroun ou l'informel à la remorque de l'État : une solution d'avenir au problème de la mobilité et de l'emploi urbain en Afrique subsaharienne », Colloque international sur le taxi organisé à Lisbonne les 20 et 21 septembre 2007 [en ligne].

KEUTCHEU J. (2015), " Le "fléau des motos-taxis". Comment se fabrique un problème public au Cameroun », Cahiers d'études africaines, n 219, pp. 509-534.

LOMME R., VIRCOULON T., (2006). «La régulation des transports informels à l'épreuve de la "guerre des taxis" collectifs en Afrique du Sud », Afrique contemporaine, $\mathrm{n}^{\circ} 218$, pp. $119-140$.

LOUVET N., ROCCI A., LE BRIS C. (2013), « L'innovation sociale, outil de renouvellement de la production de services de mobilité », Développement durable \& territoires, vol. $4, \mathrm{n}^{\circ} 3$, pp. 1-12.

MOUHOT L. (2013), Amélioration du contrôle qualité sur le réseau de bus d'Hô Chi Minh Ville, PADDI, p. 67.

NGUYEN A. (2014), " Growing taxi operator is expanding to second-tiers cities », CIMB, p. 20

NGUYEN H. Q., NGUYEN H. B., EMBEGER G. (2013), «Application of a macroscopic transport model in Ho Chi Minh City », Proceedings of the Eastern Asia Society for Transportation Studies, vol. 9, 9 p.

ONN C. C., MOHAMED R. K., SUMIANI Y. (2014), « Mode Choice between Private and Public Transport in Klang Valley, Malaysia », The Scientific World Journal, vol. 2014, p. 14.

PEREZ R., ARMAND J. (2016), « Indemniser les taxis pour révolutionner le transport des personnes en France », Terra Nova, p. 28. 
RAHMAN M. S. (2013), Integrating BRT with Rickshaws in Developing Cities: A Case Study on Dhaka City, Bangladesh, thèse de doctorat, University of Leeds.

RAllet A., AGUILERA A., GUILLOT C. (2009), « Diffusion des TIC et mobilité. Permanence et renouvellement des problématiques de recherche », Flux, Association Metropolis, $\mathrm{n}^{\circ} 78$, pp. 7-16.

ROCCI A. (2007), De l'automobilité à la multimodalité. Analyse sociologique des freins et leviers au changement de comportement vers une réduction de l'usage individuel de la voiture, thèse de doctorat, Paris 5.

SAJOUS P., THÉBERT M., NESSI H., CORALLI M. (2015), « Mobilités quotidiennes et modes d'habiter dans le périurbain francilien », Netcom, 29, 1-2, pp. 79-110.

SALLES J. (2015), « L'incertitude dans l'utilisation des médias géolocalisés : étude de deux applications reliées à la mobilité urbaine », Netcom, 29, 1-2, pp. 13-36.

SCHALEKAMP H., MCLACHLAN N. (2016), " Minibus-taxi operator reforms, engagement and attitudes in Cape Town », in Paratransit in African Cities. Operation, Regulation and Reform, London, Routledge, pp. 174-198.

SPAD. (2013), Taxi Transformation Plan, p. 81.

TUAN V. A., MATEO-BABIANO I.B. (2013), « Motorcycle taxi service in Vietnam: Its Socioeconomics Impacts and Policy Consideration », Proceedings of the Eastern Asia Society for Transportation Studies, vol. 9, p. 16.

UN-HABITAT (2013), Planning and Design for Sustainable Urban Mobility: Global Report on Human Settlements 2013, London, Routledge, p. 344.

VISIT KL (2014), « Taxi Booking Terminals To Be Installed At 14 Tourists Attractions by MyTeksi For Tourist' Convenient », communiqué de presse publié le 26 novembre 2014, p. 4.

WE ARE SOCIAL (2016), Digital in 2016 Vietnam, présentation de rapport, p. 17.

WIDMER S. (2014), « Navigation sur mesure ? Usages d'application en ville de New York », Géo Regards, n 7, pp. 55-72.

WIDMER S., PEDROZO S., KLAUSER F. (2014), « Éditorial. Mobilités et gestion des flux à l'ère numérique », Géo Regards, n 7, pp. 5-15. 


\section{ANNEXE}

Tableau 1. Entretiens réalisés auprès d'experts du transport et de l'urbanisme

\begin{tabular}{|c|c|c|c|}
\hline Date & Nom & Fonction & Organisme \\
\hline $16 / 06 / 2015$ & Norlida Abdul Hamid & Professeure associée & Universiti Teknologi MARA \\
\hline $16 / 06 / 2015$ & $\begin{array}{c}\text { Seyed Mohammadreza } \\
\text { Ghadiri }\end{array}$ & $\begin{array}{l}\text { Directeur du Department of } \\
\text { Transportation and Logistics } \\
\& \text { Supply Chain Management }\end{array}$ & $\begin{array}{c}\text { Malaysia University } \\
\text { of Science and Technology } \\
\text { (MUST) }\end{array}$ \\
\hline $18 / 06 / 2015$ & Fanny Quertamp & Directrice & PADDI \\
\hline $18 / 06 / 2015$ & Clément Musil & Chercheur associé & PADDI \\
\hline $19 / 06 / 2015$ & Thi Cam Van Nguyen & Chercheuse & $\begin{array}{c}\text { HCMC Institute } \\
\text { for Development Studies }\end{array}$ \\
\hline $23 / 06 / 2015$ & Quoc Hien Nguyen & $\begin{array}{c}\text { Doyen de la Faculté } \\
\text { d'Ingénierie du Transport }\end{array}$ & $\begin{array}{l}\text { HCMC University } \\
\text { of Transport }\end{array}$ \\
\hline
\end{tabular}

Source : auteures.

Tableau 2. Catégories de licences de taxi à Kuala Lumpur

\begin{tabular}{|c|c|c|c|c|}
\hline $\begin{array}{l}\text { Catégories de } \\
\text { services de taxi }\end{array}$ & Budget & Premium & TEKS1M & Executive \\
\hline Aire de desserte & $\begin{array}{c}\text { Centre de KL, Klang } \\
\text { Valley }^{12}\end{array}$ & Centre de KL & $\begin{array}{l}\text { Centre de KL, } \\
\text { Klang Valley }\end{array}$ & $\begin{array}{c}\text { Péninsule } \\
\text { malaisienne }\end{array}$ \\
\hline $\begin{array}{l}\text { Segments cibles } \\
\text { de mobilité }\end{array}$ & $\begin{array}{l}\text { Trajets courte } \\
\text { distance }\end{array}$ & Voyageurs aisés & Tourisme, achats & Voyageurs aisés \\
\hline Types d'opérateurs & $\begin{array}{l}\text { Entreprises } \\
\text { Particuliers }\end{array}$ & $\begin{array}{l}\text { Entreprises } \\
\text { Particuliers }\end{array}$ & Particuliers & $\begin{array}{l}\text { Entreprises } \\
\text { Particuliers }\end{array}$ \\
\hline Nombre de licences & 19085 & $\begin{array}{c}21 \\
\text { (retrait en cours) }\end{array}$ & $\begin{array}{l}291 \text { (introduction } \\
\text { en 2013) }\end{array}$ & 1904 \\
\hline $\begin{array}{c}\text { Durée de validité } \\
\text { de la licence } \\
\text { véhicule }\end{array}$ & $\begin{array}{c}\text { (neuf) } 7 \text { ans } \\
\text { (occasion) } 7 \text { ans } \\
\text { moins l'âge } \\
\text { du véhicule }\end{array}$ & - & 10 ans & $\begin{array}{c}\text { (neuf) } 7 \text { ans } \\
\text { (occasion) } 7 \text { ans } \\
\text { moins l'âge } \\
\text { du véhicule }\end{array}$ \\
\hline $\begin{array}{c}\text { Marques et modèles } \\
\text { de véhicule }\end{array}$ & $\begin{array}{c}\text { Proton Iswara, } \\
\text { Waja, ou Saga } \\
\text { BM (constructeur } \\
\text { malaisien) }\end{array}$ & - & $\begin{array}{c}\text { Proton Exora } \\
\text { (constructeur } \\
\text { malaisien) }\end{array}$ & $\begin{array}{c}\text { Berline / Minivan } \\
\text { (constructeurs } \\
\text { malaisiens } \\
\text { ou étrangers) }\end{array}$ \\
\hline $\begin{array}{l}\text { Tarif de la course } \\
\qquad(10 \mathrm{~km})\end{array}$ & $\begin{array}{c}\text { 14,25 MYR } \\
(3,5 \mathrm{USD})\end{array}$ & - & $\begin{array}{c}\text { 17,5 } \operatorname{MYR}(4,3 \\
\text { USD) }\end{array}$ & $\begin{array}{c}24,00 \mathrm{MYR} \\
(6,0 \mathrm{USD})\end{array}$ \\
\hline
\end{tabular}

Source : auteures.

12. Klang Valley est l'aire métropolitaine autour de Kuala Lumpur. 